\title{
A Reflective Learning Framework to Evaluate CME Effects on Practice Reflection
}

\author{
Kit H. Leung, PhD; Pierre Pluye, MD, PhD; Roland Grad, MD, MSc; Cynthia Weston, PhD
}

\begin{abstract}
Introduction: The importance of reflective practice is recognized by the adoption of a reflective learning model in continuing medical education (CME), but little is known about how to evaluate reflective learning in CME. Reflective learning seldom is defined in terms of specific cognitive processes or observable performances. Competencybased evaluation rarely is used for evaluating CME effects. To bridge this gap, reflective learning was defined operationally in a reflective learning framework (RLF). The operationalization supports observations, documentation, and evaluation of reflective learning performances in CME, and in clinical practice. In this study, the RLF was refined and validated as physician performance was evaluated in a CME e-learning activity.
\end{abstract}

Methods: Qualitative multiple-case study wherein 473 practicing family physicians commented on research-based synopses after reading and rating them as an on-line CME learning activity. These comments formed 2029 cases from which cognitive tasks were extracted as defined by the RLF with the use of a thematic analysis. Frequencies of cognitive tasks were compared in a cross-case analysis.

Results: Four RLF cognitive processes and 12 tasks were supported. Reflective learning was defined as 4 interrelated cognitive processes: Interpretation, Validation, Generalization, and Change, which were specified by 3 observable cognitive tasks, respectively. These 12 tasks and related characteristics were described in an RLF codebook for future use.

Discussion: Reflective learning performances of family physicians were evaluated. The RLF and its codebook can be used for integrating reflective learning into CME curricula and for developing competency-based assessment. Future research on potential uses of the RLF should involve participation of CME stakeholders.

Key Words: education, medical, continuing, reflective learning framework, reflective practice, reflective cognitive processes, reflective cognitive tasks, continuing professional development

\section{Introduction}

Reflective learning is an important component of intellectual integrity and professional practice, ${ }^{1}$ for example, self-

Disclosure: The authors report that the conduct of the presently reported study and preparation of the manuscript was supported by the Canadian Institutes of Health Research (CIHR).

Dr. Leung: Researcher, Information Technology Primary Care Research Group, Department of Family Medicine, Faculty of Medicine, McGill University; Dr. Pluye: Associate Professor, Department of Family Medicine, Faculty of Medicine, McGill University; Dr. Grad: Associate Professor, Department of Family Medicine, Faculty of Medicine, McGill University; Dr. Weston: Director, Teaching and Learning Services, Department of Educational and Counselling Psychology, McGill University.

Correspondence: Kit H. Leung, Information Technology Primary Care Research Group, Department of Family Medicine, Faculty of Medicine, McGill University, 517 Pine Avenue West, Montreal, Quebec, H2W 1S4, Canada; e-mail: kit.leung@mail.mcgill.ca.

(C) 2010 The Alliance for Continuing Medical Education, the Society for Academic Continuing Medical Education, and the Council on CME, Association for Hospital Medical Education. - Published online in Wiley InterScience (www.interscience.wiley.com). DOI: 10.1002/chp.20063 assessment in clinical practice. ${ }^{2}$ Nevertheless, little is known about how to integrate reflective learning into the curricula of continuing medical education ( $\mathrm{CME})$. The term reflective learning is seldom defined as specific observable operations. Eva and Regehr state "the term reflection is intended to indicate a conscious and deliberate reinvestment of mental energy aimed at exploring and elaborating one's understanding of the problem one has faced (or is facing) rather than aimed simply at trying to solve it." ${ }^{3}$ This statement implies that reflection and learning are inseparable actions; however, this definition does not provide an observable phenomenon; that is, "understanding" cannot be directly evaluated. In a systematic literature review, Mann et al report 2 reflective learning processes of meaning construction and interpretation. ${ }^{4}$ The report suggests that reflective learning is composed of different cognitive processes. This raises the question of how to observe occurrences of these processes.

Reflection is either not defined ${ }^{5}$ or not operationally defined. The internal validity of existing measures of reflection may be questioned, for example, the Reflection Questionnaire and the scale of Reflection-in-Learning. ${ }^{6,7} \mathrm{Re}-$ lated test items can be challenged by plausible alternative 
explanations, which inhibit respondents to make consistent answers; for example, "I often reflect on my actions to see whether I could have improved on what I did." 6 This complicates evaluation in terms of consistency of observations and adequacy in representing reflection as a theoretical construct.

Moreover, rare studies propose an operational definition of reflective learning for observing, describing, and documenting reflection performance related to $\mathrm{CME}$ activities. The effect of CME on the performance of physicians is subject to debate and skepticism. ${ }^{8}$ Participating in CME or continuing professional development activities appears to have little effect on changing physician performance. ${ }^{5,8}$ Although a competency-based evaluation for CME that involves evaluation of physician participants' competence or performance has been considered a must for more than 20 years, ${ }^{9}$ related evidence-based educational guidelines for CME were just proposed in $2009 .{ }^{8}$ Nevertheless, time on learning task is still used as a key parameter for learning outcome assessment, and for earning CME credit. Any relationship between the adopted reflective learning model of Schön in CME and a time-on-task-oriented accreditation system is not well supported by research evidence. ${ }^{10}$ This approach does not evaluate any $\mathrm{CME}$ effects on reflective competency, let alone medical practice outcomes. Developing a method for evaluating reflection, specifically, performances of pondering information received for practice in CME and any related effect on clinical practice, is an urgent need.

Thus, we operationalized reflective learning in the form of a reflective learning framework (RLF), which was refined and validated in a CME context. The operationalization aimed to describe reflective performances for observation, documentation, and evaluation of practice reflection. The research questions were

- What are the properties of reflective learning?

- Are these properties valid in a CME context?

- Can reflective learning performances be documented and evaluated?

In a literature review, we first identified properties of reflective learning in terms of cognitive processes and related cognitive tasks to form an initial RLF. After this RLF was examined in a pilot study, it was refined and validated in this full-scale qualitative multiple-case study. To address concerns around the validity of these properties, both studies were performed in an e-learning activity in CME, which involved reading, rating, and commenting on research-based synopses delivered as e-mail alerts.

\section{Methods}

\section{Building a Conceptual Framework}

We used an inductive approach to build an RLF. As reflective learning involves psychological processes, related properties were identified in terms of cognitive processes, and were specified as observable cognitive tasks via a literature review in educational psychology. These properties and their relationship were represented in an initial version of the RLF.

The concept of reflective learning originated from Dewey's reflective thought, ${ }^{1-13}$ and was seen as an equivalent to the behaviorist conception of "mediated generalization." 14 Dewey's theory of reflection influenced Schön's theory of reflective learning, and Resnick's studies of higher-order thinking.

Based on Dewey's philosophical explanation of reflective thought, we first used Schön's theory ${ }^{1,15-18}$ to define concepts of reflective learning with respect to professional development globally. Second, we specified these concepts using Resnick's higher-order thinking occurrences, ${ }^{19,20}$ and Donald's working model of higher-order learning ${ }^{21}$ in terms of cognitive processes and related tasks. Finally, after specifications were examined and modified iteratively in consultation with 3 expert educational psychologists, the initial version of the RLF was established, and presented at a CME Congress for expert feedback. ${ }^{22}$

The RLF comprised 5 processes: Meaning Construction, Interpretation, Change, Validation, and Generalization. Each process identified a level of complexity of reflective learning, and was specified by 5 observable cognitive tasks. The relationships between these processes and tasks were structured, and ordered corresponding to their complexity (see EXHIBIT 1).

\section{Validating the RLF in a Pilot Study}

The initial version of the RLF was propositional and examined in a pilot study from which a coding scheme was derived. The pilot study involved data collected from 50 cases randomly selected ${ }^{23}$ from 1766 cases (written comments on research-based synopses submitted on-line by family physicians). Cases are explained in the next section.

A deductive approach was taken. The RLF processes and cognitive tasks served as categories and subcategories, respectively, in the coding scheme. For each case, a written comment was read and assigned to the corresponding cognitive task code. Because reflective learning processes were conceptualized as interdependent, and interconnected, multiple coding was allowed. In other words, 1 part of a comment could be coded to more than 1 cognitive task.

The pilot study comprised 2 stages to limit ambiguities of the coding scheme. In these stages the agreement and disagreement of each code between independent coders were compared in terms of frequency. The first stage involved 2 independent coders who analyzed 20 of the 50 randomly selected cases. Disagreements between coders revealed ambiguous definitions of codes and overlapping cognitive tasks. This stage clarified the definitions of codes, and merged codes when needed, which led to a revised coding scheme for the next stage. 
EXHIBIT 1. Initial Version of the Reflective Learning FrameworkCognition and Description

1. Meaning construction: Frame characteristics of an idea. Composing/ structuring a rule for extracting meaning from given information, for example, "if, then" logic.

1.1. Raising a question: Ask a question(s) about given information.

1.2. Filtering information: Accept/reject information.

1.3. Organizing information: Structure information in a particular way, for example, concept mapping or sequencing.

1.4. Linking new information to prior knowledge: Connect past experience with new information.

1.5. Explaining usefulness/not usefulness: Describe what is useful/not useful for practice.

2. Interpretation: Understand meanings of an idea. Inferring, analyzing, or comparing meanings of given information.

2.1. Selecting critical component(s): Select parts or components that are relevant to the issue.

2.2. Identifying critical relationship(s): Connect critical components of an issue.

2.3. Comparing alternative ideas: Examine similarities and (or) differences of other ideas (with outside norms).

2.4. Clarifying an idea/practice: Explain an idea/practice using principles such as medical guidelines.

2.5. Making hypothesis: Make an assumption based on facts or evidence.

3. Conceptual change: Change understanding of an idea. Reformulating a perception of given information.

3.1. Making minor change: Add or discard nonessential component(s) to an idea or practice.

3.2. Making essential change: Add or discard essential component(s) to an idea or practice.

3.3. Adopting a new idea: Decide to use a new idea for practice.

3.4. Discarding an idea: Dispose of an old idea or practice.

3.5. Replacing an idea: Switch from an old idea to a new idea for practice.

4. Validation: Verify validity of ideas-ratifying accuracy and consistency of ideas.

4.1. Confirming/disconfirming an idea(s). Agree/disagree with an idea(s) such as conclusions or effects.

4.2. Justifying an idea: Provide evidence for rejecting or accepting an idea.

4.3. Appraising accuracy: Critically examine soundness and (or) flaws based on evidence.

4.4. Appraising consistency: Critically examine whether ideas are stuck together based on evidence.

4.5. Assessing an ideas(s) using new knowledge. Evaluate an idea(s) with newly learned knowledge or new information.

5. Generalization: Develop a general inference for practice. Generate a holistic idea based on specifics.

5.1. Synthesis: Join components together to form a holistic idea.

5.2. Discovering new relations: Uncover new relationships of ideas.

5.3. Bridging a knowledge gap with new ideas: Identify what the gap is in a sequence, and then fill in the gap using new ideas.

5.4. Building up a course of action: Make a plan for completing a task/ bridging a knowledge gap.

5.5. Drawing a conclusion: Summarize theses and (or) antitheses to make a decision.
Excluding the independent coder in the previous stage, the second stage involved 3 new independent coders who analyzed the remaining 30 randomly selected cases. One coder was a university professor, and 2 others were members of a research team. Their codings were recorded and compared. Agreement and disagreement between coders to 1 category were represented by 1 (agreement) and 0 (disagreement). Next, 1 of us (KHL) counted the number of agreements and disagreements. When 2 agreements (or disagreement) and 1 disagreement (agreement) to 1 category were identified, KHL counted it as an agreement or vice versa, which was flagged for discussion. KHL also compared all disagreements to her coding, and discussed them with the coders. The discussion guided further revisions of the definitions for refinement. The counting of agreements was used to calculate the intercoder agreement in terms of frequency, which was $93 \%$. Thus, the coders assigned the same category to 93\% of the observed reflective performances. This agreement rate indicates the possibility of good operational definitions and categories.

Overlapped cognitive tasks were examined, and then merged. Based on pilot-study results, the coding scheme was tested and revised, and the RLF was modified accordingly. The pilot version of the RLF comprised 5 processes, and each process was specified by 3 cognitive tasks. This version is presented in EXHIBIT 2.

\section{Validating the RLF in a Qualitative Multiple-Case Study}

Adopting a deductive approach, the RLF was validated in a qualitative multiple-case study. A case was defined as 1 family physician reading, rating, and commenting on 1 researchbased synopsis received via e-mail. The research design is presented in TABLE 1. Qualitative data were collected within a prospective observational study. ${ }^{24}$ Ethical approval was received from McGill University.

\section{Research-Based Synopses and 2 Data Sources}

The 2 data sources of this research were derived from reading, rating, and commenting on research-based synopses called InfoPOEMs, as well as related interview transcripts. Reading and rating were derived from a separate study ${ }^{25}$ in which InfoPOEMs were delivered daily by e-mail to members of the Canadian Medical Association (CMA) during a 150-day study period. Family physicians earned CME credit from the College of Family Physicians of Canada, by reading and rating InfoPOEMs with the use of the Information Assessment Method (IAM). The IAM, hyperlinked to a daily InfoPOEM (http://iam2009.pbworks.com), contained a checklist of 10 items and a text box for comments that aimed to capture the cognitive impact of that information. The free-text comments were the data source for the Comment Cases. 
EXHIBIT 2. The Pilot Version of the Reflective Learning Framework ${ }^{\mathrm{a}}$

Cognitive process 1: Meaning construction. Frame characteristics of an idea.

1.1. Asking a question(s)

1.2. Identifying relevant information

1.3. Connecting information to experience and practice

Cognitive process 2: Interpretation. Understand meanings of an idea.

2.1. Specifying important or controversial issue

2.2. Comparing with norms, research or practice

2.3. Proposing a hypothesis

Cognitive process 3 : Changes of idea or practice. Changes current practice or perception.

3.1. Revising an idea or practice.

3.2. Adopting a new idea or practice.

3.3. Replacing an idea or practice.

Cognitive process 4: Validation. Verify validity of ideas.

4.1. Agreeing with the information provided

4.2. Identifying flaws in the information provided

4.3. Assessing knowledge and practice

Cognitive process 5: Generalization. Develop a general inference for practice.

5.1. Drawing a conclusion based on research or experience

5.2. Planning to apply or looking into information provided

5.3. Applying information provided in other contexts

${ }^{\text {a}}$ Reflective learning (cognitive) processes and tasks are organized from basic to complex. A cognitive process potentially occurs when at least 1 of its tasks is performed.

For the Interview Cases, the data source was derived from interviews to examine the validity of IAM. Interviewees were family physicians or general practitioners who read and rated at least 5 InfoPOEMs. Three rated InfoPOEMs were sent to interviewees prior to each interview. After a brief introduction presenting the study, a few questions were asked about

TABLE 1. Research Design: A Multiple-Case Study

\begin{tabular}{|c|c|c|}
\hline Contexts & $\begin{array}{l}\text { 1. On-Line Free } \\
\text { Comments }\end{array}$ & $\begin{array}{l}\text { 2. Telephone Guided } \\
\text { Interviews }\end{array}$ \\
\hline Sources & $\begin{array}{l}\text { Written comments on } \\
\text { InfoPOEMs }\end{array}$ & Impacts of InfoPOEMs \\
\hline Participants & 473 family physicians & 46 family physicians \\
\hline No. of cases & 1776 & 253 \\
\hline Case definitions & $\begin{array}{l}\text { One family physician } \\
\text { reading, rating, and } \\
\text { commenting on } 1 \\
\text { synopsis via the } \\
\text { Internet }\end{array}$ & $\begin{array}{l}\text { One family physician } \\
\text { explaining why } 1 \\
\text { specific item is } \\
\text { selected in the Impact } \\
\text { Assessment for the } \\
\text { synopsis read }\end{array}$ \\
\hline
\end{tabular}

a specific research-based synopsis. Among the set of InfoPOEMs rated by interviewees, we purposefully selected a sample (maximum variation sampling) to obtain about 30 ratings of each item (see items of cognitive impact in FIGURE 2). Then, interviewees were asked, "On (read date), you rated this InfoPOEM as (read impact items under scrutiny). In what specific ways (ask all that apply, such as, 'Did this InfoPOEM improve your practice?' and/or 'Did you learn something new?,' etc)." The interview concluded with 3 general questions on the overall use, satisfaction, and concerns of interviewees with research-based synopses. Transcripts of these interviews were the data source of the Interview Cases.

The purpose of using 2 different data sources for our research was to facilitate a richer understanding of reflective learning. Nevertheless, both data sources, namely, the Comment Cases and Interview Cases, likely affected the nature of reflection. In contrast to searches that involve clinical information retrieved at the point of care, the physicians did not receive research-based synopses on e-mail in response to clinical questions about a specific patient. Nevertheless, the act of reading, rating, and commenting on research-based synopses triggered reflection on using (or not using) information for a specific patient recalled by a physician. In general, the nature of reflection was likely affected by the depth of physician memory, and by the relevance of that information to their clinical practice. In addition, in the Comment Cases, reflection likely emerged from individual-physician thought; however, for the Interview Cases, thoughts ensued from the physicians and their interaction with the interviewer. This interaction or conversation possibly triggered more complex ideas. ${ }^{26,27}$

\section{Participants}

Participants were 473 practicing family physicians who read, rated, and commented on InfoPOEMs. In addition, 40 family physicians and 6 other specialists were interviewed to explain the rationale underlying their rating of 3 specific InfoPOEMs.

According to data from the 2007 Canadian National Physician Survey, ${ }^{28,29}$ our sample of 473 practicing family physicians was similar to the population of Canadian family physicians with respect to age and gender, with 2 minor differences. FIGURE 1 compares our sample with the Canadian population in terms of age. This comparison shows that the 2 curves follow a similar pattern. Nevertheless, with respect to the 34-and-under age group, younger family physicians were more likely to participate in this specific e-learning activity.

This phenomenon is also observed in gender comparison between our sample and the population of Canadian family physicians. In our sample, $52.2 \%$ of female physicians were 44 or younger. This is higher than the national average of $45.4 \%$. Thus, our sample contained more young women than the national average. Both our sample and the population 


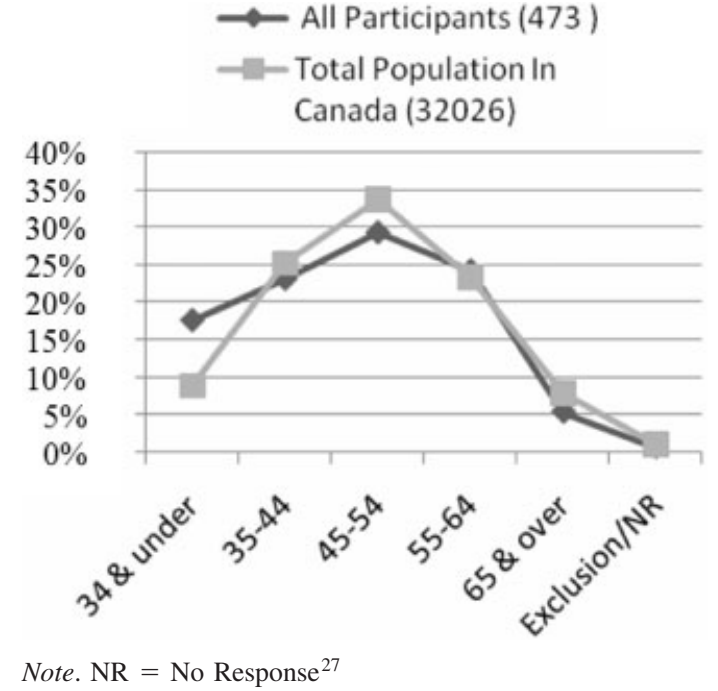

FIGURE 1. Sample and national demographics.

indicate female family physicians are younger than their male counterpart. At the national level, $45.4 \%$ female physicians are in the age groups of "34 and under" and "35 and 44," whereas $27 \%$ of male physicians are in these age groups. ${ }^{28,29}$ For our sample, the percentages are $52.2 \%$ (female physicians) versus $30.2 \%$ (male physicians). The reason for more female than male participants may derive from the age factor, because the younger generation has better computer skills for learning on-line. ${ }^{30}$

\section{Data Collection and Analysis}

As shown in TABLE 1, qualitative data were collected from 2 sources that provided 2029 cases. Specifically, 1776 comment cases derived from free-text comments, and 253 interview cases ensued from semistructured telephone interviews. An Interview Case involved 1 family physician explaining why he or she selected 1 specific item from the IAM cognitive checklist. A Comment Case involved 1 family physician reading, rating, and commenting on 1 InfoPOEM on-line (FIGURE 2).

Data analysis involved thematic analysis assisted by specialized software (MAXQDA). The analysis comprised 3 strategies: Categorization, Pattern-Matching, and Tabulation. Our unit of analysis was "Cognitive Task Extracted" (CTE). A CTE refers to a theme (a cognitive task in the RLF) that was observed and extracted from a statement(s) in a written comment or an interview transcript. The extraction involved categorization; for example, assigning a code (a cognitive task) or codes to textual data as multiple coding can be used. Then, the frequency of CTEs by RLF task was counted in a cross-case analysis.

Pattern matching involved matching the conceptualized cognitive tasks of the RLF with CTEs, which were derived from data. In a matrix, CTE frequency was matched with RLF cognitive tasks. The match indicates whether RLF tasks were relevant operations of reflective learning, and specified which tasks were supported by data. Pattern matching was performed in tabulation.

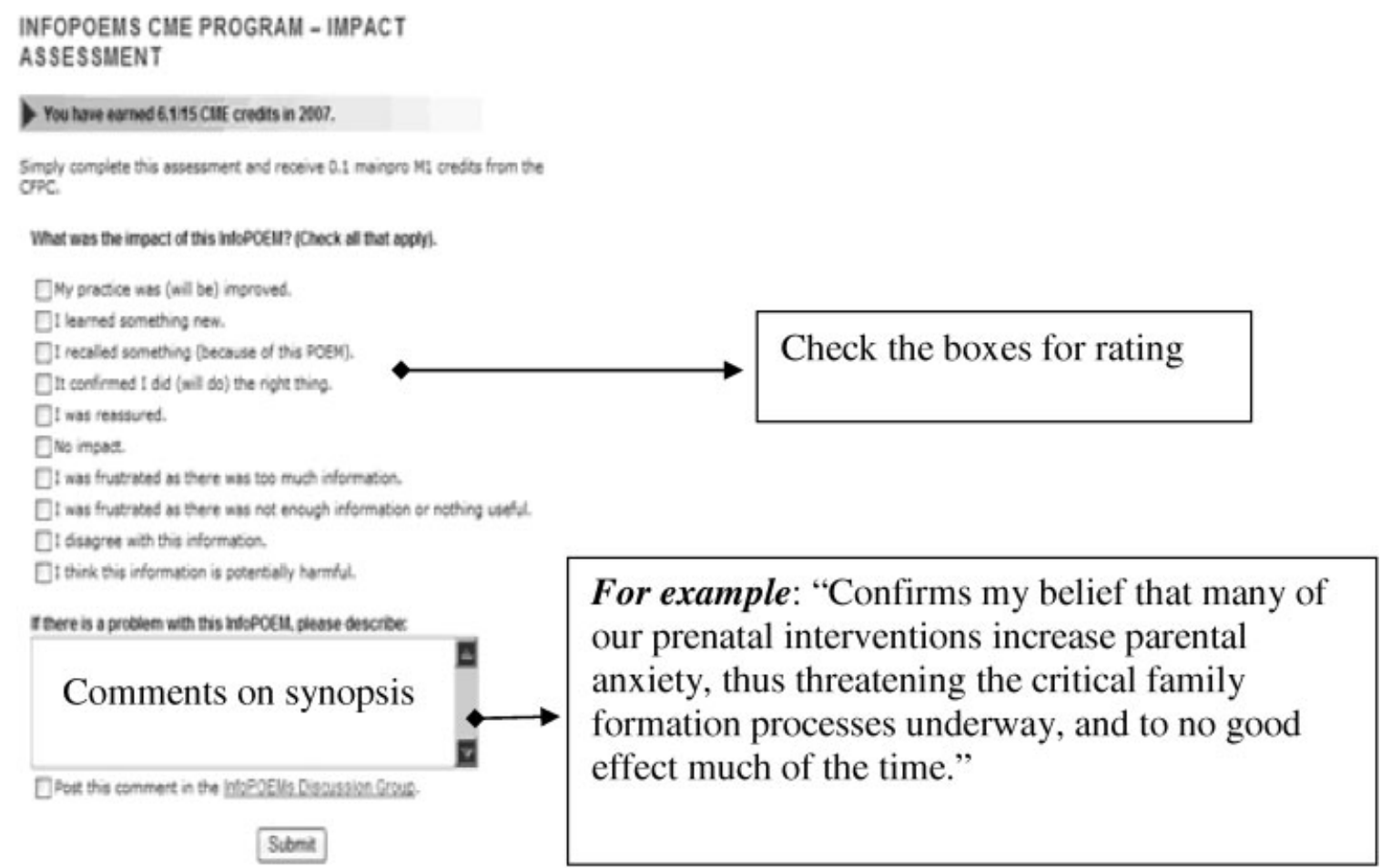

FIGURE 2. Rating and commenting on an InfoPOEM. 
Tabulation and frequency counts were used to convert qualitative data to quantitative data for comparison, and for identifying patterns in graphical displays (eg, FIGURE 1). A CTE depicted a reflective performance as it occurred; however, extracting cognitive tasks involved the possible subjective nature of the coding system. Tabulating the CTEs and frequency count of CTEs enumerated the number of times specific cognitive tasks were observed and extracted (see TABLE 2). Via comparison, inferences were made to determine (a) whether the cognitive tasks representing reflective learning occurred (or not), and (b) which cognitive processes and tasks of reflective learning were substantively aligned with the data.

For the validation of coding, $10 \%$ of the data were coded by 2 independent coders who had an educational psychology background and experience in coding. The related training session required 2 hours, and 1 additional 30-minute exercise. An extraction of 1 cognitive task from a segment, for example, 1 sentence in the text, required 30 seconds to 1 minute, depending on the complexity of the text.

The 2 coders agreed with respect to 135 cases out of $150(90 \%)$. The value of the kappa measure of agreement was 0.576 , interpreted as fair or good agreement. For each code, the agreement was above $79 \%$ (between $79 \%$ and $100 \%)$. Based on this data analysis, reflective learning processes and cognitive tasks in the RLF were either merged or substantiated.

\section{Results}

All properties of reflective learning described in the RLF were supported by the data. Each cognitive task of the RLF was extracted from these data. The results led to further revision of the RLF. This revision was based on the premise that higher-level cognitive tasks subsume lowerlevel cognitive tasks and that these form a cumulative hierarchy (eg, Bloom et al, 1956; ${ }^{31}$ Anderson and Krathwohl, $2001^{32}$ ). As such, more complex tasks involve the performance of a number of basic tasks. A supposition emerging from this study (which has yet to be researched) is that this subsuming relationship is manifested through the more frequent occurrence of basic cognitive tasks, which cumulate in more infrequent complex ones.

For example, a basic task involves the performance of a primary task, for example, "Identifying Relevant Information" (1.2, EXHIBIT 2), in reading and commenting on research-based synopses. A complex task, such as "Applying Information in Other Contexts" (5.3, EXHIBIT 2), is composed of a series of cumulative lower-level (basic) tasks. The first is to identify relevant information (1.2, EXHIBIT 2), and to compare it with norms, research, and practice (2.2, EXHIBIT 2). This information may then be agreed upon (4.1, EXHIBIT 2) via assessing knowledge and practice (4.3, EXHIBIT 2) and a conclusion drawn based on experience (5.1, EXHIBIT 2). These tasks can lead to applying information provided in another context such as sending this information to colleagues as educational material (5.3, EXHIBIT 2). This example indicates that without the performance of related basic tasks a complex task is not likely to occur. When a complex task is performed, it likely involves several basic tasks, hence the supposition that basic tasks are performed more frequently than complex tasks.

Relatively more complex cognitive tasks occurred more frequently in Interview Cases than in Comment Cases. For example, the cognitive task of "Applying Information Provided in Other Contexts" (5.3) was observed 9 (4\%) times in Comment cases, versus $46(58 \%)$ times in Interview Cases (see TABLE 2). These frequency counts suggested that the nature of the data sources possibly affected reflective performances.

In Comment Cases, the most frequently observed processes were Meaning Construction (681 CTEs, 33\%) and Interpretation (810 CTEs, 40\%). In Interview Cases, the most frequently observed processes were Meaning Construction (237 CTEs, 32.6\%) and Validation (252 CTEs, 34\%). Complex processes of Change, Validation, and Generalization were more frequently observed in Interview Cases than in Comment Cases, whereas basic processes of Meaning Construction and Interpretation were more frequently observed in Comment Cases. As a whole, complex processes were less frequently observed than basic processes in all cases (TABLE 2).

TABLE 2 indicates a decrease in the frequency of observed CTEs pertaining to complex processes, that is, a decrease from Validation $(20.3 \%)$ to Generalization $(10.6 \%)$ to Change $(3.5 \%)$. This decrease suggests Change may be the most complex level in the refined RLF.

The multiple coding illustrated both interrelations and connections between cognitive tasks; however, it also identified ambiguities. For example, taking the cognitive task of "Specifying an Important or Controversial Issue" (2.1), this task was observed and extracted from:

- "Where do I find somebody to inject botulinum?" [Case 0298 (Comment Cases); InfoPOEM Title= Botulinum might be more effective than nitroglycerine in anal fissure]

- "What is the impact of thromboprophylaxis, and why was it deemed unnecessary?" [Case 1002 (Comment Cases); InfoPOEM Title $=$ Low risk of deep venous thrombosis $(\mathrm{DVT})$ after knee arthroscopy]

As indicated in these examples, task 2.1 was often performed in the form of questioning; thus, it was also coded as "Asking a Question(s)." Because of a high intersection frequency, the latter task was merged into the former. CTE frequencies are presented in TABLE 2.

The multiple coding provided 3 analytic results, which provided data for refining the RLF. The refinement involves merging cognitive processes and tasks. The first analytic result is related to the most frequent intersection (283 times) between CTE 1.1 (Asking a Question[s]) and CTE 2.1 
Leung et al.

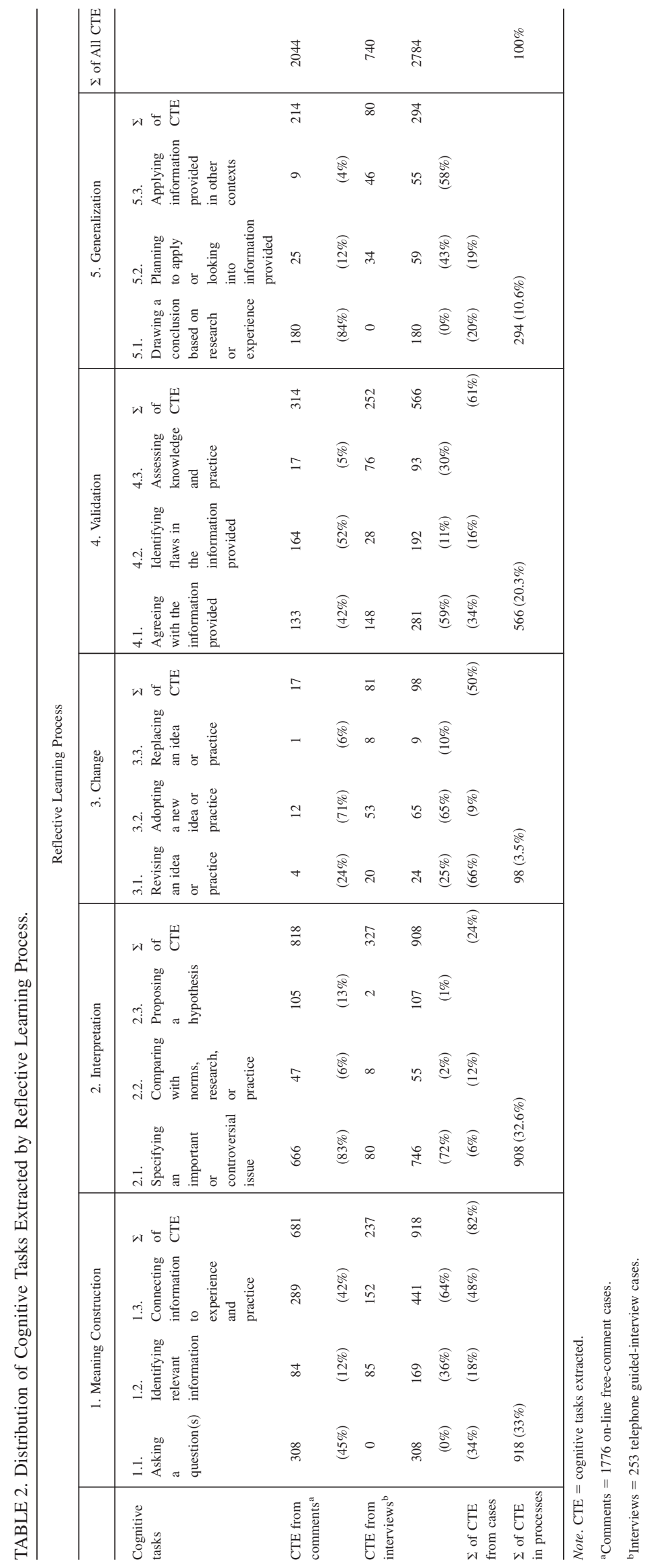

84 JOURNAL OF CONTINUING EDUCATION IN THE HEALTH PROFESSIONS-30(2), 2010 
(Specifying Important or Controversial Issue). This frequency suggests that questioning was used to specify important or controversial issues. Accordingly, CTE 1.1 was merged into CTE 2.1. Thus, CTE 2.1 was redefined as "Questioning or Specifying an Important or Controversial Issue."

The second analytic result is concerned with the frequent intersections between CTE 1.3 (Connecting Information to Experience and Practice), and all other CTEs. These interactions suggest that all RLF cognitive tasks may be performed in connection with experience and practice. Thus, CTE 1.3 was merged into all the cognitive processes whose definitions were revised accordingly.

The third analytic challenge emerged from the observation that all data coded CTE 5.1 (Drawing a Conclusion Based on Research or Experience) were also coded CTE 2.3 (Proposing a Hypothesis). This indicates that a hypothesis is often made in connection to conclusion making. Thus, CTE 2.3 was merged into CTE 5.1, and the related definition was revised.

These changes led to the integration of the process of Meaning Construction into the process of Interpretation. For hypothesis-generating purposes, the order of the $3 \mathrm{cog}$ nitive tasks of the Interpretation process was based on their frequency of occurrence. Although the most frequent task was conceived as the most basic one, the least frequent task was assumed to be the most complex. Thus, the most frequent task CTE 1.2 (169 times) was revised as CTE 1.1, the least frequent task CTE 2.2 (55 times) was revised as CTE 1.3, and amid these 2 frequencies, CTE 2.1 was revised as CTE 1.2.

\section{Discussion}

In contrast to developing a questionnaire, and using confirmatory factor analysis to validate a model, ${ }^{33-35}$ this research focuses on adequacy of representing reflective learning in a framework via observing and documenting performances that exemplify reflective practice. The refined and validated RLF aims to provide CME practitioners a framework to develop robust CME programs, and guide evaluation of practice reflection. We examine research findings for answering the following 3 research questions.

\section{Answer to the First Question}

The refined and validated RLF provides an answer to question 1: "What are the properties of reflective learning?" Reflective learning comprises 4 processes of Interpretation, Verification, Generalization, and Change. Each process can be externalized by 3 observable cognitive tasks, respectively. The term Validation was replaced by Verification to avoid any association with statistics. A short version of the refined RLF and the related codebook are described in EXHIBIT 3. Details and CTE-related illustrations are available upon request.

\section{Questions 2 and 3}

Answers to questions 2 and 3 are interdependent. The underlying assumption is that when properties of reflective learning comprise valid operations in a CME context, reflective learning performances can be observed, documented, and evaluated. The answer to question 2, "Are these

EXHIBIT 3. The Refined RLF/Codebook (Short Version)

\begin{tabular}{|c|c|}
\hline Cognitive Tasks & Definitions \\
\hline Identifying relevant information & Selecting information that is useful to address problem at hand or reduce uncertainty \\
\hline Questioning or specifying important or controversial issue & Asking questions or stating issues that are of value or subjected to controversy to practice \\
\hline Comparing with norms, research, or practice of others & $\begin{array}{l}\text { Examining similarities and (or) differences of ideas or practice using norms, research, or } \\
\text { practice of others as criteria. }\end{array}$ \\
\hline Agreeing with the information provided & Explaining an agreement with an idea or practice described in the information \\
\hline Identifying flaws in the information provided & Explaining a flawed idea or practice identified in the information provided \\
\hline Assessing one's knowledge and practice & Evaluating one's own knowledge base and experience \\
\hline Drawing a conclusion based on research or experience & $\begin{array}{l}\text { Synthesizing information with other research-based information or experience to make a } \\
\text { conclusion }\end{array}$ \\
\hline Planning to apply or looking into information provided & $\begin{array}{l}\text { Making an arrangement to use information selected, or to seek complementary } \\
\text { information from other sources }\end{array}$ \\
\hline Applying information provided in other contexts & Using information provided for other purposes \\
\hline Revising an idea or practice & $\begin{array}{l}\text { Reviewing, reorganizing, amending a current idea or practice for an update or } \\
\text { improvement }\end{array}$ \\
\hline Adopting a new idea or practice & Selecting and following a new complementary idea or practice \\
\hline Replacing an idea or practice & Switching a current practice or idea to a newly introduced one \\
\hline
\end{tabular}


properties valid in a CME context?" is yes. The properties of the RLF were validated in the context of an online individual e-learning activity. The participants were 473 practicing physicians. As indicated in FIGURE 1, the age of this sample had a pattern similar to the Canadian physician population. To be valid, the practice reflection of the sample was observed by using the revised RLF in the same context with a team of independent coders. The related validation refined the RLF, and was triangulated with 2029 cases. Thus, the properties of reflective learning as captured in the RLF should be valid. Along the same logic, the answer to question 3, "Can reflective learning performances be documented and evaluated?" is "yes." It follows that the impact of CME on practice reflection can be evaluated accordingly.

\section{Potential Use of the RLF}

One potential use of the RLF is observing and documenting the impact of CME for evaluation. In 2008, the participants at 1 workshop ${ }^{36}$ suggested the RLF can be used to integrate practice reflection in CME curricula and for assessment:

- Can use the framework to improve educational activities in our courses to facilitate reflection

- Change our evaluations to measure reflective content

- Applicable to our education curriculum delivery

Nevertheless, further research may refine and validate the proposed RLF, which might eventually be used for integrating reflective learning into CME curricula and for developing competency-based assessment.

We identify 3 advantages for integrating reflective learning in CME curricula. First, the RLF and the related codebook provide CME educators with a blueprint to specify observable and measurable objectives, and with a guide for developing competency-based assessment tools for effects of CME on practice reflection.

Second, the concrete characteristics of reflective learning defined in the codebook can be used in self-directed learning activities (eg, the Personal Learning Projects of the Royal College of Physicians and Surgeons of Canada) as prompts to induce complex reflective learning processes of Generalization and Change such as:

- What are the possible risks or flaws of using the suggested management plan? (Generalization)

- Given the above risks, what can be done to revise this management plan? (Change)

Third, the RLF can be used to observe specific performances of change, which is considered to be an important effect of CME on practice. Change is not merely a commitment or a plan to change, but can be described in specific performances in the form of revising an idea or practice via an application of research evidence. For example:
"We all have large numbers of patients who are taking protein pump inhibitors and sometimes over a long period of time. So it (this InfoPOEM) would make me more vigilant regarding such patients getting extra calcium supplementation, or having bone mineral density tests." [Case 031 (Interview Case); InfoPOEM Title= Long-term Proton pump inhibitors (PPI) use increases hip fracture risk]

The example indicates that performances of change can be described, and then observed for evaluation and accreditation using competency-based assessment.

In clinical practice, the RLF potentially assists physicians' recognition of cognitive tasks performed in the context of their self-directed learning, and stimulates their reflection on action. In addition, the RLF codebook can be used to observe and document reflective practice in research. The time needed for coding text using this codebook will depend on the length of the text and coding experience of coders. A detailed tutorial will assist nonexperienced coders without a specialized educational background to do coding. Certainly, the potential use of the RLF is subjected to exploration and research.

The present study faces several limitations. First, our deductive explanatory approach with a pilot framework constrained the interpretation of cases, and limited the potential discovery of other properties of reflective learning compared to an inductive exploratory approach. Second, our study was performed in a specific context, that is, on-line reading, rating, and commenting on research-based synopses, and the generalization to other educational contexts should be taken with caution. Third, qualitative case studies are usually based on multiple sources of evidence (eg, archives, documents, interviews, and observations), whereas we had access to a limited set of data (free comments and interviews). Fourth, in terms of levels of complexity, ordering cognitive processes by frequency of occurrence must be considered as a supposition to be examined in future research. In addition, the formulation of the RLF was guided by the literature that contributed to the development process, and no new items emerged from the data. Thus, further empirical research on the RLF may indicate new properties of reflective learning, which were not captured in our literature review, and not suggested by our data.

In conclusion, despite these limitations, the RLF provides a comprehensive definitive portrait of reflective learning in the form of observable performances. The validated RLF and the related codebook are tools to observe and document reflection in CME, clinical practice, and research. CME educators can use the refined RLF and the observation codebook for integrating reflective learning into curricula, and for designing instructional strategies in accordance with these objectives. Future research should examine potential uses of the RLF, for example, "To what extent does applying the RLF to CME provide a workable framework to support practice reflection?" This should involve a quantitative approach, and the participation of CME stakeholders, such as policy makers and practicing physicians. 


\section{Lessons for Practice}

\section{- For research on reflective practice in pa- tient care, the RLF provides specifications of reflection that can be observed, docu- mented, and evaluated.}

- Specifications of cognitive tasks assist physicians to perform and record reflective practice in self-assessment and self-directed learning.

- The RLF provides educators a blueprint to construct reflective learning curricula in which the effects of CME on practice reflection are observable and measurable.

\section{Acknowledgments}

Robert Bracewell and Alain Breuleux (Faculty of Education, McGill University), Gary Boyd (Faculty of Education, Concordia University), Janique Johnson-Lafleur and Yuejing Meng (Information Technology Primary Care Research Group, Family Medicine, McGill University) all contributed to this work. We are grateful to Dr. Allen Shaughnessy, PharmD (Department of Family Medicine, Tufts University) for reviewing the manuscript, and for his insightful constructive comments. Early on, this study was supported by a grant from the Social Sciences and Humanities Research Council of Canada. In a later stage, the study was supported by a scholarship awarded by the Fonds pour la formation de chercheurs et l'aide à la recherche (Fonds FCAR), Québec, Canada. In the final stage, it was supported by a grant from the Canadian Institutes of Health Research (CIHR). Pierre Pluye holds a New Investigator Award from the Canadian Institutes of Health Research (CIHR). For their research, Pierre Pluye and Roland Grad are supported by CIHR, the Fonds de Recherche en Santé du Québec (FRSQ), the Department of Family Medicine, McGill University, and the Herzl Family Practice Center. This study was approved by the Ethics Committee of McGill University. The Information Assessment Method mentioned in this article is protected by registered copyrights (2005): No. CA 1031348 "A Scale to Assess the Impact of Information Hits Derived from Electronic Resources," and No. CA 1032304 "Une Echelle pour Evaluer L'Impact des Informations Issues de Ressources Electroniques," and registered copyrights (2008): No. CA 1057518 "A Scale to Assess the Cognitive Impact, Relevance and Use of Information hits Derived from Electronic Resources," and No. CA 1057519 "Une Échelle Pour Évaluer L'Impact Cognitif, la Pertinence et L'Utilisation des Informations Issues de Ressources Électroniques.”

\section{References}

1. Schön DA. The Reflective Practitioner. New York, NY: Basic Books; 1983.

2. Silver I, Campbell C, Marlow B, Sargeant J. Self-assessment and continuing professional development: the Canadian perspective. J Contin Educ Health Prof. 2008;28(1):25-31.

3. Eva KW, Regehr G. "I'll never play professional football" and other fallacies of self-assessment. J Contin Educ Health Prof. 2008;28(1): 14-19.

4. Mann K, Gordon J, MacLeod A. Reflection and reflective practice in health professions education: a systematic review 2007. http:// www.springerlink.com/content/a226806k3n5115n5/fulltext.pdf. Accessed June 1, 2008.

5. Horsley T, O'Neill J, Campbell C. The quality of questions and use of resources in self-directed learning: personal learning projects in the maintenance of certification. J Contin Educ Health Prof. 2009;29(2):91-97.

6. Kember D, Leung YP, Jones A et al. Development of a questionnaire to measure the level of reflective thinking. Assess Eval Higher Educ. 2000;25(4):381-398.

7. Sobral DT. Medical students' reflection in learning in relation to approaches to study and academic achievement. Med Teach. 2001; 23(5):508-513.

8. Davis D, Galbraith R. Effectiveness of continuing medical education: American College of Chest Physicians evidence-based educational guidelines. http://www.chestjournal.org/content/135/3_suppl/42S. full.html. Accessed September 16, 2009.

9. Cohen G, Davis D. Continuing medical education and the competent family physician. Can Fam Phys. 1986;32:348-351.

10. Pluye P, Grad RM, Leung KH et al. Brief self-directed e-learning activities using information delivery and retrieval technology in a continuing medical education context: a literature review, an environmental scan, and interviews with CME experts. McGill Fam Med Online Stud. Vol. 4, 2009. Available at http://mcgill-fammedstudies-recherchemed fam.pbworks.com/MFMSO200904e01. Accessed January 1, 2010.

11. Dewey J. Experience and nature: nature, communication and meaning. In: Hickman L, Alexander TM, eds. The Essential Dewey. Vol 2. Bloomington, IN: Indiana University Press; 1998:50-66.

12. Dewey J. How We Think: A Restatement of the Relation of Reflective Thinking to the Educative Process. Boston, MA: D.C. Heath; 1933.

13. Dewey J. The theory of inquiry. In Hickman L, Alexander TM, eds. The Essential Dewey. Vol 2. Bloomington, IN: Indiana University Press; 1998:169-179.

14. Tolman EC. A new formula for behaviorism. Psychological Review 1922;29:44-53. http://psychclassics.yorku.ca/Tolman/formula.htm. Accessed January 1, 2007.

15. Schön DA. Beyond the Stable State. New York, NY: Random House; 1971.

16. Schön DA. Educating the Reflective Practitioner: Toward a New Design for Teaching and Learning in the Professions. San Francisco, CA: Jossey-Bass; 1987.

17. Schön DA. The theory of inquiry: Dewey's legacy to education. Curric Inq. 1992;22(2):119-139.

18. Schön DA, Rein M. Frame reflection. Acad Manag Rev. 1996;21(2): 576-579.

19. Resnick LB. Education and Learning to Think. Washington, DC: National Academies Press; 1987.

20. Resnick LB. Instruction and the cultivation of thinking. In: Entwistle N, ed. Handbook of Educational Ideas and Practices. New York, NY: Routledge; 1990;694-707.

21. Donald JG. Learning to Think: Disciplinary Perspective. San Francisco, CA: Jossey-Bass; 2002.

22. Leung KH, Pluye P, Roland G, Marrow B, Weston C. A new conceptual framework for reflective learning in CME. In: CME Congress, May 31, 2008.

23. Rand Corporation. Probability Functions. A Million Random Digit with 100,000 Normal Deviates. Glencoe, IL: The Free Press; 1955. 
24. Grad R, Pluye P, Mercer J, et al. Impact of research-based synopses delivered as daily e-mail: a prospective observational study. J Am Med Inform Assoc. 2008;15(2):240-245.

25. Pluye P, Grad R. M, Repchinsky C, et al. IAM: A comprehensive and systematic information assessment method for electronic knowledge resources. In: Dwivedi A, ed. Handbook of Research on IT Management and Clinical Data Administration in Healthcare. Hershey, PA: IGI Publishing. In press.

26. Vygotsky LS. Educational psychology. Silverman R, trans. Boca Raton, FL: CRC Press; 1997 (original work published 1926).

27. Leung KH. Conversation theory: online discussion and course design. In: Luppicini R, ed. Handbook of Conversation Design for Instruction Application. Hershey, PA: Information Science Reference; 2008: 107-123.

28. National Physician Survey: National Demographics. http://www.nation alphysiciansurvey.ca/nps/2007_Survey/Results/ENG/Provincial/pdf/ Prov.to.Can/CANADA\%20RESPONSE\%20RATE\%20Demographics. pdf. Accessed August 15, 2008.

29. National Physician Survey: Results for Family Physicians/General Practitioners. http://www.nationalphysiciansurvey.ca/nps/2007_Survey/
Results/ENG/FP/Sex\%20\&\%20Age/Q4/Q4_CORE.only.pdf. Accessed August 15, 2008.

30. Brown JS. Growing up digital: how the Web changes work, education, and the ways people learn. Change. 2000; March/April, 10-20.

31. Bloom MD, Englehart MD, Furst EJ, Hill WH, Krathwohl DR. Taxonomy of Educational Objectives: Cognitive Domain. New York, NY: McKay; 1956.

32. Anderson LW, Krathwohl DR, eds. A Taxonomy for Learning, Teaching, and Assessing: A Revision of Bloom's Taxonomy of Educational Objectives. New York, NY: Longman. 2001.

33. Mamede S, Schmidt H. Correlates of reflective practice in medicine. Adv Health Sci Educ. 2005;10(4):327-337.

34. Mamede S, Schmidt HG. The structure of reflective practice in medicine. Med Educ. 2004;38(12):1302-1308.

35. Mamede S, Schmidt HG, Penaforte JC. Effects of reflective practice on the accuracy of medical diagnoses. Med Educ. 2008;42(5):468-475.

36. Leung KH, Pluye P, Grad RM. Application of a framework to promote practice reflection. In Sixth Annual Continuing Professional Development (CPD) Accredited Providers Conference. October 3rd, 2008, Ottawa. 